\title{
Community-level educational attainment and dementia: a 6-year longitudinal multilevel study in Japan
}

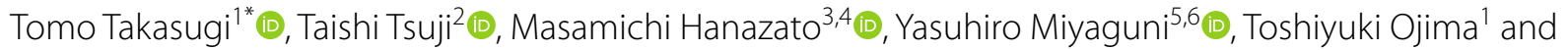
Katsunori Kondo 3,6 (1)

\begin{abstract}
Background: As the understanding of the association between community-level education and dementia is insufficient, this study examined the contextual association of community-level prevalence of low educational attainment on the risk of dementia incidence. With this study, we further explored the potential differences in the aforementioned associations for urban and non-urban areas.

Methods: We analyzed 6 years of prospective cohort data from the Japan Gerontological Evaluation Study, beginning with the baseline data collected between 2010 and 2012, for 51,186 physically and cognitively independent individuals aged $\geq 65$ years (23,785 men and 27,401 women) from 346 communities in 16 municipalities across 7 prefectures. We assessed dementia incidence using available data from the long-term care insurance system in Japan. We dichotomized education years as $\leq 9$ and $\geq 10$ years and aggregated individual-level educational attainment as a community-level independent variable. Model 1 covariates were age and sex. Income, residential years, disease, alcohol, smoking, social isolation, and population density were added in Model 2. We conducted multiple imputation to address the missing data. We performed a two-level (community and individual) survival analysis to calculate hazard ratios (HRs) and 95\% confidence intervals (Cls).
\end{abstract}

Results: The results indicate that the cumulative incidence of dementia during the follow-up period was $10.6 \%$. The mean proportion with educational attainment of $\leq 9$ years was $40.8 \%$ (range: 5.1-87.3\%). Low community-level educational attainment was significantly associated with higher dementia incidence (HR: 1.04; 95\% Cl: 1.01-1.07), estimated by 10 percentage points of low educational attainment after adjusting for individual-level educational years and covariates. While the association was significant in non-urban areas (HR: 1.07; 1.02-1.13), there was no association in urban areas (HR: 1.03; 0.99-1.06).

Conclusions: Older people living in communities with low educational attainment among their age demographic develop dementia more often compared with those living in areas with high educational attainment after adjusting for individual-level educational attainment and covariates; the association was pronounced in non-urban areas. Securing education for adolescents as a life course and population approach could thus be crucial in preventing dementia later in life among older people living in non-urban areas.

\footnotetext{
*Correspondence: takasugi@hama-med.ac.jp

${ }^{1}$ Department of Community Health and Preventive Medicine, Hamamatsu University School of Medicine, 1-20-1 Handayama, Higashi-ku, Hamamatsu, Shizuoka 431-3192, Japan

Full list of author information is available at the end of the article
} permitted by statutory regulation or exceeds the permitted use, you will need to obtain permission directly from the copyright holder. To view a copy of this licence, visit http://creativecommons.org/licenses/by/4.0/. The Creative Commons Public Domain Dedication waiver (http://creativecommons.org/publicdomain/zero/1.0/) applies to the data made available in this article, unless otherwise stated in a credit line to the data. 
Keywords: Socioeconomic status, Education, Cognitive decline, JAGES cohort, Multilevel analysis

\section{Background}

Dementia is a major global health issue caused by rapidly aging populations worldwide, with which over 50 million people are currently affected. The total number of people with dementia is expected to reach 82 million by 2030 and 152 million by 2050 [1]. Further, half of the 10 million new dementia cases occur annually in Asia [2]. Dementia not only affects individuals' personal and family lives and careers but also carries enormous medical and social care costs [3].

Although there is no cure for dementia, a recent review reported that $40 \%$ of dementia cases are preventable; the authors stated that attention should be paid to the following 12 risk factors: low education in early life, hearing loss, traumatic brain injury, hypertension, alcohol consumption, obesity in midlife, smoking, depression, social isolation, physical inactivity, air pollution, and diabetes in late life; the second-highest percentage of these risk factors is less education [4]. A systematic review showed that low education is associated with increased cognitive decline or dementia [5]. Recent studies have begun emphasizing the association between community-level education, rather than the individual level, and dementia and cognitive impairment. For example, it is possible to examine how community-level education inequality affects individual-level dementia incidence after adjusting for individual-level education. A cross-sectional study found that low community-level education is associated with declining cognition [6]. However, some studies have found associations between area of residence and dementia, such as a reduction in cognitive decline among adults living in urban areas compared with rural areas [7, 8]. Meanwhile, a recent U.S. study found significantly lower dementia prevalence among adults living in rural areas compared with urban areas over the past few decades, which can be attributed to improvements in educational attainment [9]. In short, explorations of the relationship between community-level educational attainment and dementia among urban and rural older adults have been insufficient $[9,10]$.

The aim of this longitudinal study using a large-sample data set was to examine the risk of dementia among older populations living in communities with higher proportions of low educational attainment, defined as $\leq 9$ years, compared with the risk in communities with lower proportions of low education attainment. We performed multilevel (community and individual) analyses to investigate the contextual associations of community-level educational attainment on developing dementia among older individuals after we adjusted for confounding factors. Additionally, we explored the potential differences in associations between community-level education and dementia for urban and non-urban areas.

\section{Methods \\ Participants}

This research was a 6-year prospective longitudinal study using data from a large sample acquired from the Japan Gerontological Evaluation Study (JAGES). One objective of JAGES is identifying social and behavioral factors associated with dementia onset among physically and cognitively independent individuals aged $\geq 65$ years $[11$, 12]. For the survey, a baseline mail self-reported questionnaire was administered from August 2010 to January 2012. In the study, 95,827 older people were chosen by random sampling from 16 municipalities in 7 prefectures in Japan, including urban and non-urban areas. Among 62,426 respondents (response rate: 65.1\%), 56,687 had valid information in terms of ID number, sex, and age (valid response rate: 59.2\%). Of the 56,687 participants with valid responses, 54,539 (96.2\%) were successfully linked to the records of dementia incidence during a 6-year follow-up term. A total of 51,186 participants (23,785 men and 27,401 women) were available for the present multilevel survival analyses (Fig. 1). This sample size was determined after excluding 3353 respondents who either lived in communities with fewer than 30 respondents or had limitations in basic activities of daily living, such as walking, bathing, and toileting, to ensure that the sample was both functionally and cognitively independent. Our research protocol and informed consent method were approved by the Human Subjects Committees of Nihon Fukushi University (no. 10-5) and the Chiba University Faculty of Medicine (no. 2493). All methods were carried out in accordance with Declaration of Helsinki.

\section{Measures \\ Dependent variable}

Our outcome variable was dementia incidence based on each municipality's publicly available long-term care insurance records. Japan's Ministry of Health, Labor and Welfare (MHLW) established a scale called the Degree of Independency in Daily Lives of Demented Individuals (hereafter "dementia scale") [13], and each local government sent personal investigators to participants' homes to assess their eligibility for nursing care, such as need for home helpers. The investigators evaluated physical 


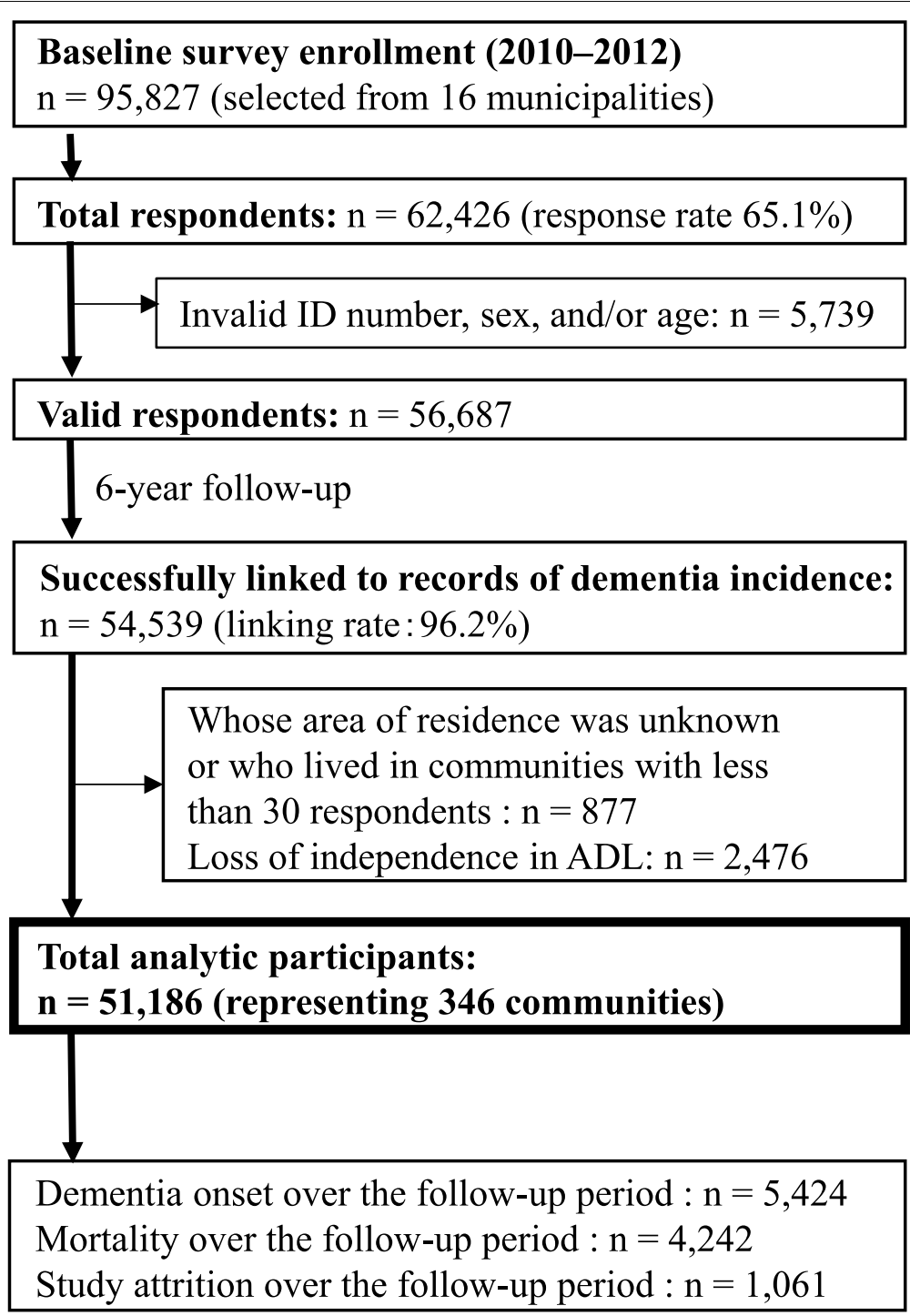

Fig. 1 Flow of participants in the cohort study

function, daily life ability, cognitive function, mental and behavioral disorders, adaptation to social life, and special medical treatment within 14 days [14, 15]. Following the assessment, the investigators classified the participants into one of eight ranks on the dementia scale according to their cognitive disability (Supplementary Table S1) $[15,16]$.

The dementia scale correlated highly with the Minimental State Examination (Spearman rank correlation $\rho=-0.74$ ) [17]. Moreover, another study, where neuropsychiatrists conducted clinical interviews as defined by the International Psychogeriatric Association, found that the dementia scale was an effective indicator for the clinical diagnosis. Sensitivity and specificity were 73 and $96 \%$ for rank II dementia [18]. According to MHLW, we defined rank II dementia or above as showing some symptoms, behaviors, or communication problems during daily lives $[15,19]$. We discontinued data tracking participants who did not develop dementia before they died or were lost to follow-up during the 6-year study period.

Community- and individual-level independent variables Educational attainment was measured with the question, "How many years of formal education have you had?" with the following response options: <6, 6-9, 10-12, and $\geq 13$ years. For our study, we dichotomized the variable as $\leq 9$ years or $\geq 10$ years [20]. We set the cutoff at 
9 years because 9 years of education has been compulsory in Japan since 1947 [21]. We aggregated individual-level educational attainment within the community to be a community-level independent variable.

\section{Covariates}

We evaluated a number of risk factors for dementia as potential confounders in this study that had been identified in earlier research [4, 22]. Age at baseline and sex were distributed based on the municipalities, and we coded age as a continuous variable between 65 and 103 . For the community-level covariate, we divided habitable areas' population density in the participants' residential school districts into quartiles $(\geq 10,100,7900-10,099$, $3280-7899$, or $<3280$ persons per square kilometer) [23]. Then, we calculated equivalized household income by dividing household income by the square root of the number of household members, and we grouped annual income into one of the three categories: $\leq 1.99,2-3.99$, or $\geq 4$ million yen [19]. Years of residence were grouped into one of the seven categories: $<5,5-9,10-19,20-29$, $30-39,40-49$, or $\geq 50$ years. Current medical treatment for diseases known to increase dementia risk (stroke, hypertension, diabetes, and/or hearing loss) [4] was collected with a "yes/no" choice for each disease. For health behaviors, we measured frequency of alcohol consumption (every day, 3-6 days/week, 1-2 days/week, 1-3 days/ month, $\leq 1 \mathrm{day} /$ month) and smoking status (non, past, or current). Further, we asked about the number of household members living with the participants and how frequently the participants met friends and acquaintances. Social isolation has been defined as social contact with other people less than once a month [4], whereas for our study, we defined social isolation as living alone and meeting with others less than once a month [23].

\section{Classification of urban and non-urban areas}

We used the European Union (EU) and Organisation for Economic Co-operation and Development (OECD) definition of a functional urban area (FUA). An FUA combines multiple municipalities and comprises a city and its surroundings with less densely populated local units that are part of the commuting areas, and FUAs are excellent for comparing socioeconomic status (SES) among cities [24]. FUAs are classified into the following four categories: 1) small urban area: population below 200,000; 2) medium-sized urban area: population 200,000-500,000; 3) metropolitan area: population 500,000 to 1.5 million; and 4) large metropolitan area: population above 1.5 million [25]. For this study, we set the cutoff population for an urban area at 500,000 because most economic activity in Japan is concentrated in metropolitan and large metropolitan areas [25]. Therefore, we grouped metropolitan and large metropolitan areas into the category of urban area and categorized all other areas as non-urban [26]. By this division, 5 municipalities were urban areas and 11 were non-urban.

\section{Statistical analyses}

We conducted multiple imputation based on multivariate normal imputation to address the missing data [27]. Specifically, we used 20 imputed data sets for analysis, with inferences for the regression coefficients acquired by merging the results over the imputed data sets applying Rubin's rules [28]. The imputed model contained dementia onset, educational attainment, income, social isolation, years of residence, disease status, and health behaviors. We performed a multilevel survival mixedeffects parametric analysis incorporating individualand community-level factors. Because a recent research reported that community-level factors had different impacts on urban and non-urban areas, we modeled the two separately [9]. Then, we calculated the hazard ratio (HR) and the 95\% confidence interval (CI) for developing dementia during the follow-up term. The HR of community-level educational attainment was estimated as a 10 percentage point difference in aggregated educational attainment [23]. We included community- and individual-level educational attainment and cross-level interaction terms in the crude model and assessed whether the contextual association of community depended on individual-level attributes. Thereafter, we added individual-level covariates (age and sex) in Model 1 and added the community-level covariate and other individual-level covariates (income, years of residence, stroke, hypertension, diabetes, hearing loss, frequency of drinking, smoking status, and social isolation) in Model 2. We used STATA/MP 17 (Stata Corp., College Station, TX) for all statistical analyses.

\section{Results}

The 51,186 participants in this study contributed 267,383 person-years. The median follow-up period was 2120 days, with an interquartile range of 251 . In the study, 5424 participants $(10.6 \%)$ acquired dementia during the follow-up period, with an incidence per 1000 personyears of 20.3 people. There were 4242 and 1061 fatalities and losses to attrition, respectively (Fig. 1). Table 1 presents all respondents' demographics and socioeconomic characteristics stratified by urban versus non-urban areas. Overall, most participants in non-urban areas had an educational attainment of $\leq 9$ years and an equivalized income of $\leq 1.99$ million yen. We calculated the mean (SD) and range for proportion of educational attainment of $\leq 9$ years in each community, which was $40.8 \%$ (17.1\%) and $5.1-87.3 \%$, respectively (Table 2 ). 
Table 1 Descriptive Statistics of All Individual-level Variables

\begin{tabular}{|c|c|c|c|c|c|c|}
\hline \multirow[b]{2}{*}{ Individual-level variables } & \multicolumn{2}{|l|}{ Urban } & \multicolumn{2}{|c|}{ Non-urban } & \multicolumn{2}{|l|}{ Total } \\
\hline & $\mathbf{n}$ & $\%$ & $\mathbf{n}$ & $\%$ & $\mathbf{n}$ & $\%$ \\
\hline Total & 30,219 & $59.0 \%$ & 20,967 & $41.0 \%$ & 51,186 & $100.0 \%$ \\
\hline \multicolumn{7}{|l|}{ Dementia onset } \\
\hline No-dementia & 27,239 & $90.1 \%$ & 18,523 & $88.3 \%$ & 45,762 & $89.4 \%$ \\
\hline Dementia & 2980 & $9.9 \%$ & 2444 & $11.7 \%$ & 5424 & $10.6 \%$ \\
\hline \multicolumn{7}{|c|}{ Educational attainment (year) } \\
\hline Less than 9 & 12,865 & $42.6 \%$ & 12,394 & $59.1 \%$ & 25,259 & $49.3 \%$ \\
\hline 10 or longer & 17,320 & $57.3 \%$ & 8545 & $40.8 \%$ & 25,865 & $50.5 \%$ \\
\hline Other and data missing & 34 & $0.1 \%$ & 28 & $0.1 \%$ & 62 & $0.1 \%$ \\
\hline \multicolumn{7}{|l|}{ Sex } \\
\hline Male & 14,295 & $47.3 \%$ & 9490 & $45.3 \%$ & 23,785 & $46.5 \%$ \\
\hline Female & 15,924 & $52.7 \%$ & 11,477 & $54.7 \%$ & 27,401 & $53.5 \%$ \\
\hline \multicolumn{7}{|l|}{ Age } \\
\hline $65-74$ & 17,943 & $59.4 \%$ & 11,642 & $55.5 \%$ & 29,585 & $57.8 \%$ \\
\hline $75-84$ & 10,622 & $35.2 \%$ & 7814 & $37.3 \%$ & 18,436 & $36.0 \%$ \\
\hline$\geqq 85$ & 1374 & $4.5 \%$ & 1791 & $8.5 \%$ & 3165 & $6.2 \%$ \\
\hline \multicolumn{7}{|l|}{ Equivalized income (yen) } \\
\hline Less than 1,999,999 & 14,065 & $46.5 \%$ & 11,815 & $56.3 \%$ & 25,880 & $50.6 \%$ \\
\hline $2,000,000-3,999,999$ & 12,211 & $40.4 \%$ & 7078 & $33.8 \%$ & 19,290 & $37.7 \%$ \\
\hline $4,000,000$ or higher & 3694 & $12.2 \%$ & 1869 & $8.9 \%$ & 5563 & $10.9 \%$ \\
\hline Data missing & 248 & $0.8 \%$ & 205 & $1.0 \%$ & 453 & $0.9 \%$ \\
\hline \multicolumn{7}{|l|}{ Years of residence } \\
\hline Less than 5 & 373 & $1.2 \%$ & 477 & $2.3 \%$ & 850 & $1.7 \%$ \\
\hline $5-9$ & 430 & $1.4 \%$ & 597 & $2.8 \%$ & 1028 & $2.0 \%$ \\
\hline 10-19 & 1074 & $3.6 \%$ & 1185 & $5.7 \%$ & 2259 & $4.4 \%$ \\
\hline $20-29$ & 1463 & $4.8 \%$ & 1165 & $5.6 \%$ & 2629 & $5.1 \%$ \\
\hline $30-39$ & 2797 & $9.3 \%$ & 1823 & $8.7 \%$ & 4620 & $9.0 \%$ \\
\hline $40-49$ & 5745 & $19.0 \%$ & 2986 & $14.2 \%$ & 8731 & $17.1 \%$ \\
\hline 50 or longer & 18,315 & $60.6 \%$ & 12,712 & $60.6 \%$ & 31,027 & $60.6 \%$ \\
\hline Data missing & 21 & $0.1 \%$ & 21 & $0.1 \%$ & 43 & $0.1 \%$ \\
\hline \multicolumn{7}{|l|}{ Social isolation } \\
\hline No & 29,317 & $97.0 \%$ & 20,503 & $97.8 \%$ & 49,820 & $97.3 \%$ \\
\hline Yes & 852 & $2.8 \%$ & 429 & $2.0 \%$ & 1280 & $2.5 \%$ \\
\hline Data missing & 50 & $0.2 \%$ & 35 & $0.2 \%$ & 85 & $0.2 \%$ \\
\hline \multicolumn{7}{|l|}{ Disease status in treatment } \\
\hline Stroke: Yes & 331 & $1.1 \%$ & 291 & $1.4 \%$ & 622 & $1.2 \%$ \\
\hline Hypertension: Yes & 11,965 & $39.6 \%$ & 8553 & $40.8 \%$ & 20,518 & $40.1 \%$ \\
\hline Diabetes: Yes & 5132 & $17.0 \%$ & 3364 & $16.0 \%$ & 8496 & $16.6 \%$ \\
\hline Hearing loss: Yes & 1986 & $6.6 \%$ & 1679 & $8.0 \%$ & 3665 & $7.2 \%$ \\
\hline \multicolumn{7}{|l|}{ Frequency of drinking } \\
\hline Every day & 8824 & $29.2 \%$ & 5861 & $28.0 \%$ & 14,685 & $28.7 \%$ \\
\hline 3-6 days/week & 10,917 & $36.1 \%$ & 7605 & $36.3 \%$ & 18,522 & $36.2 \%$ \\
\hline 1-2 days/week & 5017 & $16.6 \%$ & 3547 & $16.9 \%$ & 8565 & $16.7 \%$ \\
\hline $1-3$ days/month & 3039 & $10.1 \%$ & 2112 & $10.1 \%$ & 5151 & $10.1 \%$ \\
\hline Less than 1 day/month & 1540 & $5.1 \%$ & 1188 & $5.7 \%$ & 2728 & $5.3 \%$ \\
\hline Data missing & 881 & $2.9 \%$ & 654 & $3.1 \%$ & 1536 & $3.0 \%$ \\
\hline \multicolumn{7}{|l|}{ Smoking status } \\
\hline Non & 17,236 & $57.0 \%$ & 12,751 & $60.8 \%$ & 29,987 & $58.6 \%$ \\
\hline Past & 9612 & $31.8 \%$ & 6003 & $28.6 \%$ & 15,615 & $30.5 \%$ \\
\hline
\end{tabular}


Table 1 (continued)

\begin{tabular}{|c|c|c|c|c|c|c|}
\hline \multirow[b]{2}{*}{ Individual-level variables } & \multicolumn{2}{|c|}{ Urban } & \multicolumn{2}{|c|}{ Non-urban } & \multicolumn{2}{|l|}{ Total } \\
\hline & $\mathbf{n}$ & $\%$ & $\mathbf{n}$ & $\%$ & $\mathbf{n}$ & $\%$ \\
\hline Current & 3235 & $10.7 \%$ & 2101 & $10.0 \%$ & 5336 & $10.4 \%$ \\
\hline Data missing & 136 & $0.4 \%$ & 113 & $0.5 \%$ & 248 & $0.5 \%$ \\
\hline
\end{tabular}

Table 3 shows the results of the multilevel survival analyses for incident dementia. As per the analysis results for all participants, Model 2-including the communitylevel covariate and individual-level covariates as well as community-level high prevalence of low educational attainment-demonstrated a significant relationship with dementia risk (HR: 1.04; 95\% CI: 1.01-1.07), estimated by 10 percentage points of increment of a proportion of educational attainment in a community area. Individuallevel educational attainment showed a significant association with dementia probability (HR: 1.08; 95\% CI: 1.01-1.16 in Model 2). However, there were no significant cross-level interaction terms in Models 1 and 2.

In Model 2, among participants living in non-urban areas, community-level high prevalence of low educational attainment showed a statistically significant

Table 2 Descriptive Statistics of Community-level Variables

\begin{tabular}{|c|c|c|c|c|c|c|}
\hline \multirow[b]{2}{*}{ Community-level Variables } & \multicolumn{2}{|c|}{ Urban } & \multicolumn{2}{|c|}{ Non-urban } & \multicolumn{2}{|c|}{ Total } \\
\hline & $\mathbf{n}$ & $\%$ & $\mathbf{n}$ & $\%$ & $\mathrm{n}$ & $\%$ \\
\hline Total & 292 & $84.4 \%$ & 54 & $15.6 \%$ & 346 & $100.0 \%$ \\
\hline \multicolumn{7}{|c|}{ Proportion of Educational attainment } \\
\hline Mean (SD) & & $40.8 \%$ & $(17.1 \%)$ & & & \\
\hline (Min.-Max.) & & $(5.1-87.3 \%)$ & & & & \\
\hline \multicolumn{7}{|c|}{ Population density (persons per square $\mathrm{km}$ of inhabitable area) } \\
\hline Highest quartile $(\geq 10,100)$ & & 86 & & & & \\
\hline Second quartile $(7900-10,099)$ & & 87 & & & & \\
\hline Third quartile (3280-7899) & & 86 & & & & \\
\hline Lowest quartile $(<3280)$ & & 87 & & & & \\
\hline
\end{tabular}

Table 3 Multilevel Survival Analysis for Developing Dementia Onset (Participants Nested in 346 Community Areas)

\begin{tabular}{|c|c|c|c|c|c|c|c|c|c|}
\hline & \multicolumn{2}{|c|}{ Crude } & & \multicolumn{2}{|c|}{ Model 1} & & \multicolumn{2}{|c|}{ Model 2} & \\
\hline & HR & $95 \% \mathrm{Cl}$ & & HR & $95 \% \mathrm{Cl}$ & & HR & $95 \% \mathrm{Cl}$ & \\
\hline \multicolumn{10}{|l|}{ All participants $(n=51,186)$} \\
\hline Community-level educational attainment ${ }^{a}$ & 1.04 & $(1.02-1.07)$ & * & 1.03 & $(1.01-1.05)$ & * & 1.04 & $(1.01-1.07)$ & * \\
\hline Individual-level educational attainment ${ }^{\mathrm{b}}$ & 1.54 & $(1.43-1.65)$ & * & 1.11 & $(1.03-1.19)$ & * & 1.08 & $(1.01-1.16)$ & * \\
\hline Cross-level interaction & 0.96 & $(0.92-0.99)$ & * & 0.98 & $(0.94-1.01)$ & & 0.98 & $(0.94-1.02)$ & \\
\hline \multicolumn{10}{|l|}{ Non-Urban $(n=20,967)$} \\
\hline Community-level educational attainment ${ }^{a}$ & 1.08 & $(1.03-1.13)$ & * & 1.06 & $(1.01-1.11)$ & * & 1.07 & $(1.02-1.13)$ & * \\
\hline Individual-level educational attainment ${ }^{b}$ & 1.67 & $(1.44-1.93)$ & * & 1.10 & $(0.95-1.28)$ & & 1.07 & $(0.92-1.25)$ & \\
\hline Cross-level interaction & 0.92 & $(0.87-0.98)$ & * & 0.98 & $(0.91-1.05)$ & & 0.98 & $(0.92-1.05)$ & \\
\hline \multicolumn{10}{|l|}{ Urban $(n=30,219)$} \\
\hline Community-level educational attainment ${ }^{a}$ & 1.01 & $(0.98-1.04)$ & & 1.02 & $(0.99-1.05)$ & & 1.03 & $(0.99-1.06)$ & \\
\hline Individual-level educational attainment ${ }^{b}$ & 1.51 & $(1.39-1.64)$ & * & 1.11 & $(1.02-1.21)$ & * & 1.09 & $(1.00-1.18)$ & \\
\hline Cross-level interaction & 0.97 & $(0.91-1.03)$ & & 0.98 & $(0.93-1.04)$ & & 0.98 & $(0.93-1.04)$ & \\
\hline
\end{tabular}

Notes: $\mathrm{HR}$ hazard ratio, $\mathrm{Cl}$ confidence interval; ${ }^{\mathrm{a}} \mathrm{HR}$ for 10 percentage point increment of proportion of educational attainment in a community area; ${ }^{\mathrm{b}} \mathrm{HR}$ for educational attainment less than $9 \mathrm{yr}$. Model 1: crude model + age + sex; Model 2: Model $1+$ community-level covariate (population density) + individual-level covariates (income, years of residence, stroke, hypertension, diabetes, hearing loss, frequency of drinking, smoking status, and social isolation). *: $p<0.05$ 
relationship with dementia risk (HR: 1.07; 95\% CI: $1.02-$ 1.13). We found a statistically significant cross-level interaction term (HR: 0.92; 95\% CI: $0.87-0.98$ in the crude model) such that individuals with low educational attainment showed $8 \%$ lower dementia incidence, estimated by 10 percentage points of increment of a proportion of educational attainment in a community area. For the participants living in urban areas, the HRs of communitylevel high prevalence of low educational attainment were not significant in any of the models (HR: 1.03; 95\% CI: 0.99-1.06 in Model 2). In urban areas, individual-level educational attainment showed a significant relationship with a high likelihood of developing dementia (HR: 1.11; 95\% CI: 1.02-1.21 in Model 1), but the significance disappeared in Model 2.

\section{Discussion}

This study is the first to reveal contextual associations of community-level prevalence of low educational attainment on developing dementia among older people using longitudinal and large-scale sample data. Older adults living in communities with a higher prevalence of low educational attainment among their age demographic tended to develop dementia more often than those living in areas with a lower prevalence of low educational attainment after adjusting for individual-level educational attainment and covariates. The association was pronounced in non-urban areas.

Our results support the systematic review showing consistent evidence of an association between lower community-level SES indicators, such as the proportion of adult population with no high school degree, and declining cognition after personal SES factors are controlled for. A few longitudinal studies included in the review show that community-level SES was significantly associated with cognitive decline. Social isolation is proposed to be a mediator between low communitylevel SES and declining cognition [22]. Further, previous studies have also proposed years of residence $[7,22]$ as a potential confounder. In our models, possible mediators and confounding factors, such as the abovementioned, were fully adjusted to the limitations of previous studies. The current study was a longitudinal study with 50,000 participants, although the systematic review mainly included small cross-sectional studies of fewer than 10,000 participants [22]. Therefore, our study design was more robust than that of prior studies.

We found that the contextual association of community-level prevalence of low educational attainment on dementia incidence. People living in communities with low education among adults might depend much more on community-level rather than individual-level resources due to limitations in residents' individual-level resources. This means that poor-quality community-level resources can affect community residents' quality of life and health. Some disadvantages of low community-level SES associated with low education communities (poorly maintained walkways, parks, shopping areas, and neighborhood organizations) were associated with lower cognitive decline [6]. Moreover, previous studies have found that low food store availability and low sidewalk installation is associated with increased dementia incidence [15, 29]. In the current study, this lack of physical and social resources in communities with lower education was associated with dementia incidence compared to communities with higher education.

Bridging social capital, which indicates the connection between different groups or SES levels, can be less developed in communities with low education than in high-education communities because of limited local resources. Therefore, disseminating information and action might be inactive in communities with less education [30]. For example, people living in communities with stronger bridging social capital might quickly acquire health or dementia prevention information through various local networks, an idea called "social contagion" [30, 31]. Moreover, the concept of informal social control shows that people with higher social capital work harder to maintain social order; such individuals ask people with unhealthy lifestyles, who include people with low education, to change their health behaviors. Additionally, people with unhealthy lifestyles can observe correct health behaviors and imitate their actions through community networks [30,31]. Therefore, improving the bridging of social capital among older adults with lower education could be associated with decreased dementia risk, which is attributable to the associations of social contagion and informal social control.

Conversely, there is strong bonding social capital in communities with high proportion of low educational attainment; people with less education survive and help each other. However, bonding social capital is associated with more psychological disorders [30], which can be associated with dementia risk. Additionally, because communities with less community-level education have more sparse resources than communities with more education, social capital might have some negative impacts, such as placing excessive demands on members. Limited local resources lead to increased pain for a few limited, reliable members of a community and exclusion of newcomers with few years of residence [30]. Generally, because the negative impacts of social capital might be associated with a higher risk of dementia, it is necessary to increase social capital in communities with less educational attainment by developing human and economic resources, such as training community leaders and 
volunteers; these efforts will build community resources to reduce the negative impacts of social capital [30].

Our study is consistent with review articles that found that people in urban centers tended to develop dementia less than those in rural areas [10, 32]. Studies have found unequal distributions of resources related to better cognitive function (health clinics, bookstores, and libraries) to be associated with lower demands for such resources in less educated communities along with a smaller tax base to pay for the resources [6]. This situation is more pronounced in non-urban areas than in urban areas.

People living in communities with less education, especially non-urban areas, could be disproportionately exposed to chronic and stressful life conditions that generate hazards, such as few employment opportunities and low incomes. This situation results in a lack of social resources (social clubs and neighborhood organizations) and safe physical resources, including gyms [6]. In the current study, the limited resources in non-urban areas compared with those in urban areas could be associated with the increased incidence of dementia.

In one systematic review with a meta-analysis, early life rural living was strongly associated with a risk of Alzheimer's disease [32]. Additionally, previous studies have found greater educational inequality in rural areas, such as insufficient educational opportunities and poorer quality of education [10,33]. Although some children left rural areas and continued their studies in urban areas [33], many participants in this study might have long lived in the same rural areas where they had received their education [34]. We did not clarify the quality of education or the childhood residential areas in our study; however, educational disadvantage in rural areas could be associated with greater risk of dementia [34]. Conversely, in a U.S. study, cognitive decline was more significant among adults living in rural areas compared with urban areas in recent decades. This association reflects narrowing rural-urban disparities, which are attributable to increasing education between 1910 and 1940 [9]. The same trend could occur in Japan in the future given that compulsory education was extended from six to nine years in 1947 [21].

This study has some limitations. First, we did not identify transfers of residential areas, including urban to non-urban areas or vice versa. Additionally, we did not identify participants' childhood residential areas. However, we did adjust for years of residence to overcome the limitations of prior studies [7, 22]. Second, though we did not consider the quality of education, majority of SES indicators in the existing studies were educational years [22]. Third, we lacked data on some potentially community-level confounding factors, such as accessibility of local services, but we considered population density.
Fourth, because we did not consider the study survey response rate, there could have been selection bias in our findings. Specifically, because the respondents were healthier than non-respondents, the identified associations might be underestimated. Lastly, we failed to perform a competing risk analysis after conducting multiple imputation with a multilevel model, which could have led to bias related to not considering the competing analysis.

\section{Conclusions}

Older people living in communities with high prevalence of low educational attainment among their age demographic develop dementia more often than those living in areas with lower prevalence of low educational attainment after adjusting for individual-level educational attainment and covariates. Although the association was pronounced in non-urban areas, the identified associations were not strong. These findings establish the foundation for future studies. We do conclude, however, that securing education for adolescents as a life course and population approach might be crucial to preventing dementia later in life among older people living in nonurban areas.

\section{Abbreviations \\ Cl: Confidence interval; EU: European Union; FUA: Functional Urban Area; HR: Hazard ratio; JAGES: Japan Gerontological Evaluation Study; MHLW: Ministry of Health, Labor and Welfare; OECD: Organisation for Economic Co-operation and Development; SES: Socioeconomic status.}

\section{Supplementary Information}

The online version contains supplementary material available at https://doi. org/10.1186/s12877-021-02615-x.

Additional file 1: Supplementary Table S1. Criteria of Levels of Cognitive Disability in Japanese Long-Term Care Insurance System. The Ministry of Health, Labor and Welfare in Japan classified eight ranks on a dementia scale according to people's cognitive disability.

\section{Acknowledgments}

The authors would like to thank the study participants and the JAGES team for data collection. We would also like to thank members of the JAGES group for their advice regarding this study. We would especially like to thank Dr. Jun Aida for giving us helpful advice about statistical analyses.

\section{Authors' contributions}

TTa: conception, design, analysis and interpretation of the data, and writing the article; TTs: conception, design, interpretation of the data, and critical revision of the article; $\mathrm{MH}$ : conception, design, and critical revision of the article; YM: conception, design, and critical revision of the article; TO: critical revision of the article; and KK: conception, design, critical revision of the article, and principal investigator for the JAGES project. All authors read and approved the final draft submitted.

\section{Funding}

This study used data from JAGES (the Japan Gerontological Evaluation Study), which was supported by MEXT (Ministry of Education, Culture, Sports, Science and Technology-Japan)-Supported Program for the Strategic Research Foundation at Private Universities (2009-2013), JSPS (Japan Society for the 
Promotion of Science) KAKENHI Grant Numbers (JP18390200, JP22330172, JP22390400, JP23243070, JP23590786, JP23790710, JP24390469, JP24530698, JP24683018, JP25253052, JP25870573, JP25870881, JP26285138, JP26882010, JP15H01972, 21 K21187), Health Labour Sciences Research Grants (H22-ChojuShitei-008, H24-Junkanki [Seishu]- Ippan-007, H24-Chikyukibo-Ippan-009, H24-Choju-Wakate-009, H25-Kenki-Wakate-015, H25-Choju-Ippan-003, H26Irryo-Shitei-003 [Fukkou], H26-Choju-Ippan-006, H27-Ninchisyou-Ippan-001, H28-choju-Ippan-002, H28- Ninchisyou-Ippan-002, H30-Kenki-Ippan-006, H30-Junkankitou-Ippan-004, 21LA2003), Japan Agency for Medical Research and development (AMED) (JP17dk0110017, JP18dk0110027, JP18Is0110002, JP18le01 10009), the Research Funding for Longevity Sciences from National Center for Geriatrics and Gerontology (24-17, 24-23, 29-42, 30-22). The views and opinions expressed in this article are those of the authors and do not necessarily reflect the official policy or position of the respective funding organizations.

\section{Availability of data and materials}

Data are from the JAGES study. All enquiries are to be addressed at the data management committee via e-mail: dataadmin.ml@jages.net. All JAGES datasets have ethical or legal restrictions for public deposition due to inclusion of sensitive information from the human participants. Following the regulation of local governments that cooperated on our survey, the JAGES data management committee has imposed the restrictions upon the data.

\section{Declarations}

\section{Ethics approval and consent to participate}

The research protocol of JAGES and informed consent method were approved by the Human Subjects Committees of Nihon Fukushi University (no. 10-5) and the Chiba University Faculty of Medicine (no. 2493). We obtained written consent from the participants.

\section{Consent for publication}

Not applicable.

\section{Competing interests}

The authors declare that they have no competing interests.

\section{Author details}

${ }^{1}$ Department of Community Health and Preventive Medicine, Hamamatsu University School of Medicine, 1-20-1 Handayama, Higashi-ku, Hamamatsu, Shizuoka 431-3192, Japan. ${ }^{2}$ Faculty of Health and Sport Sciences, University of Tsukuba, 3-29-1 Otsuka, Bunkyo-Ward, Tokyo 112-0012, Japan. ${ }^{3}$ Center for Preventive Medical Sciences, Chiba University, 1-33 Yayoi-cho, Inage-Ward, Chiba City, Chiba 263-8522, Japan. ${ }^{4}$ Design Research Institute, Chiba University, 1-19-1 Bunka, Sumida-ku, Tokyo 131-0044, Japan. ${ }^{5}$ Faculty of Social Welfare, Nihon Fukushi University, Okuda, Mihama-cho, Chita-gun, Aichi 470-3295, Japan. ${ }^{6}$ Center for Gerontology and Social Science, National Center for Geriatrics and Gerontology, 7-430 Morioka-cho, Obu-City, Aichi 474-8511, Japan.

Received: 10 June 2021 Accepted: 9 November 2021

Published online: 23 November 2021

\section{References}

1. Alzheimer's Disease International: World Alzheimer Report 2019. 2019. https://www.alz.co.uk/research/WorldAlzheimerReport2019.pdf. Accessed 2 March 2021.

2. Alzheimer's Disease International: World Alzheimer Report 2015. 2015. https://www.alz.co.uk/research/WorldAlzheimerReport2019.pdf. Accessed 2 March 2021.

3. World Health Organization: Demetia: key facts. 2020. https://www.who. int/news-room/fact-sheets/detail/dementia. Accessed 2 March 2021.

4. Livingston G, Huntley J, Sommerlad A, Ames D, Ballard C, Banerjee S, et al. Dementia prevention, intervention, and care: 2020 report of the lancet commission. Lancet Commissions. 2020;396(10248):413-46.
5. Harrison SL, Sajjad A, Bramer WM, Ikram MA, Tiemeier H, Stephan BCM. Exploring strategies to operationalize cognitive reserve: a systematic review of reviews. J Clin Exp Neuropsychol. 2015;37(3):253-64.

6. Wight RG, Aneshensel CS, Miller-Martinez D, Botticello AL, Cummings JR, Karlamangla AS, et al. Urban neighborhood context, educational attainment, and cognitive function among older adults. Am J Epidemiol. 2006;163(12):1071-8

7. Xiang YX, Zare H, Guan CL, Gaskin D. The impact of rural-urban community settings on cognitive decline: results from a nationally-representative sample of seniors in China. BMC Geriatr. 2018;18:323.

8. Wu F, Guo YF, Zheng Y, Ma WJ, Kowal P, Chatterji S, et al. Social-economic status and cognitive performance among Chinese aged 50 years and older. PLoS One. 2016;11(11):e0166986.

9. Weden MM, Shih RA, Kabeto MU, Langa KM. Secular trends in dementia and cognitive impairment of US rural and urban older adults. Am J Prev Med. 2018;54(2):164-72.

10. Robbins RN, Scott T, Joska JA, Gouse H. Impact of urbanization on cognitive disorders. Curr Opin Psychiatry. 2019;32(3):210-7.

11. Kondo K, Rosenberg M, \& World Health Organization: advancing universal health coverage through knowledge translation for healthy ageing: lessons learnt from the Japan gerontological evaluation study. 2018. https:// apps.who.int/iris/handle/10665/279010. Accessed 2 Mar 2021.

12. Kondo K. Progress in aging epidemiology in Japan: the JAGES project. J Epidemiol. 2016;26(7):331-6.

13. Tamiya N, Noguchi H, Nishi A, Reich MR, Ikegami N, Hashimoto H, et al. Population ageing and wellbeing: lessons from Japan's long-term care insurance policy. Lancet. 2011;378(9797):1183-92.

14. Ministry of Health, Labor and Welfare: Textbook for Expert Investigators Concerning a Certification of Needed Long-term Care, Rvised Edition 2009. 2009. https://www.mhlw.go.jp/file/06-Seisakujouhou-12300000Roukenkyoku/0000077237.pdf. Accessed 2 Mar 2021.

15. Tani Y, Suzuki N, Fujiwara T, Hanazato M, Kondo K. Neighborhood food environment and dementia incidence: the Japan Gerontological evaluation study cohort survey. Am J Prev Med. 2019;56(3):383-92.

16. Hikichi H, Kondo K, Takeda T, Kawachi I. Social interaction and cognitive decline: results of a 7-year community intervention. Alzheimers Dement. 2017;3(1):23-32.

17. Hisano $\mathrm{S}$. The relationship between revised Hasegawa dementia scale (HDS-R), Mini-mental state examination (MMSE) and bed-fast scale, dementia scale. Jpn J Geriatr Psychiat. 2009;20(8):883-91.

18. Noda H, Yamagishi K, Ikeda A, Asada T, Iso H. Identification of dementia using standard clinical assessments by primary care physicians in Japan. Geriatr Gerontol Int. 2018;18(5):738-44.

19. Takasugi T, Tsuji T, Nagamine Y, Miyaguni Y, Kondo K. Socioeconomic status and dementia onset among older Japanese: a six-year prospective cohort study from the Japan Gerontological evaluation study. Int J Geriatr Psychiatry. 2019;34:1642-50.

20. Basta NE, Matthews FE, Chatfield MD, Brayne C, Mrc C. Community-level socio-economic status and cognitive and functional impairment in the older population. Eur J Pub Health. 2008;18(1):48-54.

21. Ministry of Education, Culture, Sports, Science and Technology: Overview: Principles Guide Japan's Educational System. https://www.mext.go.jp/en/ policy/education/overview/index.htm. Accessed 2 March 2021.

22. Besser LM, McDonald NC, Song Y, Kukull WA, Rodriguez DA. Neighborhood environment and cognition in older adults: a systematic review. Am J Prev Med. 2017;53(2):241-51.

23. Tsuji T, Kanamori S, Miyaguni Y, Hanazato M, Kondo K. Community-level sports group participation and the risk of cognitive impairment. Med Sci Sports Exerc. 2019;51(11):2217-23.

24. Dijkstra, L., Poelman, H., \& Veneri, P.: OECD Regional Development Working Papers 2019/11: The EU-OECD definition of a functional urban area 2019. https://doi.org/10.1787/d58cb34d-en.

25. OECD: Redefining "urban": a new way to measure metropolitan areas. 2012. https://doi.org/10.1787/9789264174108-en. Accessed 2 March 2021.

26. Ide K, Tsuji T, Kanamori S, Jeong S, Nagamine Y, Kondo K. Social participation and functional decline: a comparative study of rural and urban older people, using Japan Gerontological evaluation study longitudinal data. Int J Environ Res Public Health. 2020;17(2):617. 
27. Lee KJ, Carlin JB. Multiple imputation for missing data: fully conditional specification versus multivariate Normal imputation. Am J Epidemiol. 2010;171(5):624-32.

28. Rubin DB. Multiple imputation after $18+$ years. J Am Stat Assoc. 1996;91(434):473-89.

29. Tani Y, Hanazato M, Fujiwara T, Suzuki N, Kondo K. Neighborhood sidewalk environment and incidence of dementia in older Japanese adults: the Japan Gerontological evaluation study cohort. Am J Epidemiol. 2021:190(7):1270-80.

30. Berkman LF, Kawachi I, Glymour MM. Social epidemiology. 2nd ed. New York: Oxford University Press; 2014

31. Murayama H, Miyamae F, Ura C, Sakuma N, Sugiyama M, Inagaki H, et al. Does community social capital buffer the relationship between educational disadvantage and cognitive impairment? A multilevel analysis in Japan. BMC Public Health. 2019;19:1442.

32. Russ TC, Batty GD, Hearnshaw GF, Fenton C, Starr JM. Geographical variation in dementia: systematic review with meta-analysis. Int J Epidemiol. 2012;41(4):1012-32.

33. Hendrie HC, Smith-Gamble V, Lane KA, Purnell C, Clark DO, Gao SJ. The Association of Early Life Factors and Declining Incidence Rates of dementia in an elderly population of African Americans. J Gerontol Ser B Psychol Sci Soc Sci. 2018;73:S82-9.

34. Saenz JL, Downer B, Garcia MA, Wong R. Cognition and context: ruralurban differences in cognitive aging among older Mexican adults. J Aging Health. 2018;30(6):965-86.

\section{Publisher's Note}

Springer Nature remains neutral with regard to jurisdictional claims in published maps and institutional affiliations.

- fast, convenient online submission

- thorough peer review by experienced researchers in your field

- rapid publication on acceptance

- support for research data, including large and complex data types

- gold Open Access which fosters wider collaboration and increased citations

- maximum visibility for your research: over $100 \mathrm{M}$ website views per year

At BMC, research is always in progress.

Learn more biomedcentral.com/submissions 